\title{
Estado nutricional de adolescentes do Maranhão, Brasil, por critérios nacional e internacional
}

\author{
Nutritional status of adolescents in the state of Maranhão, Brazil, \\ assessed by national and international criteria
}

Andréa Suzana Vieira Costa ${ }^{1}$

Maria Bethânia da Costa Chein ${ }^{1}$

Sueli Rosina Tonial ${ }^{1}$

Mônica Elinor Alves Gama ${ }^{1}$

Maylla Luanna Barbosa Martins ${ }^{1}$

Carlos Leonardo Figueiredo Cunha ${ }^{1}$

Diego Salvador Muniz da Silva ${ }^{2}$

Paulo Roberto Silva Cruz ${ }^{2}$

Luciane Maria Oliveira Brito

\footnotetext{
${ }^{1}$ Programa de PósGraduação em Saúde Materno-Infantil, Centro de Ciências Biológicas e da Saúde, Universidade Federa do Maranhão. Pç. Gonçalves Dias $21 / 2^{\circ}$, Centro. 65.020240 São Luís MA Brasil. lucianebrito@ufma.br ${ }^{2}$ Faculdade de Medicina, Centro de Ciências Biológicas e da Saúde, Universidade Federal do Maranhão.
}

\begin{abstract}
This study sought to compare national and international criteria for assessing the nutritional status of adolescents. A cross-sectional analytical study was conducted in the period from July 2007 to January 2008 with a representative sample comprised of 1256 adolescents from the state of Maranhão. Body mass index (BMI) for age and gender was used to diagnose underweight, normal weight and overweight, using the criteria proposed by Conde and Monteiro and the World Health Organization (WHO). Chi-square, McNemar concordance and Spearman correlation tests were applied. According to the criteria of Conde and Monteiro and the WHO, there were significant differences among the boys with respect to low weight and obesity. It was observed that there was no significant divergence between the two criteria, and a significant positive correlation (0.011) between the two criteria was detected. With this analysis it can be seen that there are many divergences between the criteria used, therefore the best option and the advantage of using one or the other cannot be singled out. However, it should be stressed that the national criterion can also be used more, since there are no significant differences with the criteria advocated by the Ministry of Health of the WHO.

Key words Nutritional status, Adolescents, Body mass index
\end{abstract}

Resumo O presente estudo objetivou comparar os critérios nacional e internacional para avaliação do estado nutricional de adolescentes. Realizou-se um estudo transversal e analítico, no período de julho de 2007 a janeiro de 2008, com uma amostra representativa do estado do Maranhão composta por 1.256 adolescentes. O indice de massa corporal (IMC) por gênero e idade foi utilizado para diagnosticar baixo peso, peso adequado e excesso de peso, empregando-se os critérios propostos por Conde e Monteiro e pela Organização Mundial de Saúde (OMS). Foram aplicados testes qui-quadrados, concordância de McNemar e correlação de Spearman. Segundo os critérios de Conde e Monteiro e da OMS, houve diferenças significativas entre os meninos quanto ao baixo peso e obesidade. Observou-se que não houve discordância significativa entre os dois critérios. Constatou-se uma correlação positiva e significativa $(0,011)$ entre os dois critérios. Com esta análise pode-se perceber que não há muitas discordâncias entre os critérios utilizados, portanto não podemos apontar o melhor critério e as vantagens de se utilizar um ou outro. Contudo, vale ressaltar que o critério nacional também pode ser mais utilizado, já que não tem diferenças significativas com aquele preconizado pelo Ministério da Saúde, da OMS.

Palavras-chave Estado nutricional, Adolescentes, Indice de massa corporal 


\section{Introdução}

O uso de medidas antropométricas para avaliação nutricional tem sido muito utilizado em estudos populacionais, principalmente entre os adolescentes que apresentam certa dificuldade na sua avaliação em função da variabilidade do crescimento e das dimensões corporais que dependem da idade, gênero e maturação sexual. Embora com limitações, é considerado o modo mais prático e de menor custo ${ }^{1-5}$.

A avaliação nutricional de crianças, jovens, adultos e idosos é um instrumento de muita utilidade na saúde pública. A medida do crescimento na infância, por meio do uso de gráficos, é um dos mais importantes indicadores básicos para avaliar o bem-estar de crianças, individualmente ou coletivamente. Contudo, parece haver unanimidade sobre a importância da utilização de referência ou padrão de crescimento para avaliar e acompanhar a situação nutricional da criança e de populaçõos s,6,7. $^{2}$.

A utilização de referência de crescimento para avaliar o estado nutricional de crianças não é recente. Desde os anos 70, quando os gráficos de crescimentos começaram a ser amplamente utilizados, tem havido constantes debates sobre a necessidade de desenvolver curvas específicas para cada país ou sobre a possibilidade de se aceitar universalmente uma única referência. Em 1978, a Organização Mundial de Saúde (OMS) passou a recomendar a referência $\mathrm{NCHS} / \mathrm{CDC}^{8}$ como padrão internacional. Apesar da recomendação ter sido motivo de várias discussões, pois apresentava uma série de limitações relacionadas à origem e ao tipo de dados, tais limitações tornaram essa referência inadequada para a avaliação do crescimento $^{2,6,9,10}$.

Com o reconhecimento dos problemas da curva de crescimento da NCHS/WHO, utilizada como referência internacional, em 1995 a OMS iniciou discussão sobre a necessidade de construção de uma nova para crianças e adolescentes que levasse em consideração alguns aspectos como o aleitamento materno, a inclusão de outros indicadores antropométricos, a utilização de dados de outros países e não só dos EUA, entre outros. Em 2006 a OMS apresenta as novas curvas de crescimento que incluem dados antropométricos de peso, estatura, perímetro cefálico e braquial e dobras cutâneas tripcipital e subescapular ${ }^{2,6,9}$.

O estado nutricional dos menores de 20 anos tradicionalmente vem sendo classificado com base em critérios probabilísticos. Em 1997, a Internacional Obesity Task Force (IOTF), indicou o
IMC como medida mais razoável para fins de comparação de estudos internacionais sobre prevalência de sobrepeso e obesidade em adolescentes. Apesar das recomendações, o uso do IMC em adolescentes ainda é um tema bastante controverso ${ }^{2}$.

Em 2000, foram divulgadas as curvas de crescimento revisadas pelo Center for Disease Control and Prevention (CDC), nas quais foi incluída a curva de percentis de IMC para idade de 2 a 20 anos. Cole et al. ${ }^{11}$ divulgaram uma curva baseada em dados populacionais obtidos em estudos realizados em seis países (Brasil, Reino Unido, Hong-kong, Holanda, Singapura e Estados Unidos), nesta proposta, com base em um critério estatístico, determinou-se para cada sexo e idade entre 2 a 18 anos, qual o valor de IMC que corresponderia ao de $25 \mathrm{Kg} / \mathrm{m}^{2}$ (para identificar sobrepeso) e $30 \mathrm{Kg} / \mathrm{m}^{2}$ (para identificar obesidade) aos 18 anos de idade ${ }^{2,12}$.

Conde e Monteiro ${ }^{1}$ elaboraram curvas brasileiras do IMC de crianças e adolescentes com base nos dados da Pesquisa Nacional sobre Saúde e Nutrição (PNSN), utilizando metodologia similar à de Cole et al. ${ }^{11}$, e os valores para sobrepeso são aproximadamente uma unidade de IMC menores do que na curva internacional. Estudos revelam a sensibilidade ligeiramente mais elevada, para os valores críticos de IMC proposto por Conde e Monteiro ${ }^{1}$, que resultou em diferença de $10 \%$ no número de resultados falso-negativos em comparação aos demais critérios, principalmente quando comparados ao IOTF, Must et al. citado por Abrantes et al. ${ }^{13}$, WHO, nos sujeitos do sexo masculino e a IOTF, nos do feminino ${ }^{2,14}$.

Apesar da existência de uma referência internacional para monitorar o crescimento e o desenvolvimento da criança e adolescente, não há consenso a respeito das validades das referências internacionais para o uso em países em desenvolvimento, nos quais as crianças e os adolescentes têm substancialmente menores valores de IMC e maturam mais tarde do que as populações que refletem referências internacionais. Os estudos têm discutido a necessidade de utilizar pontos de corte específicos para cada população devido às diferenças nos valores do IMC. Com a proposição do novo critério para o diagnóstico de excesso de peso entre adolescentes brasileiros, de acordo com o IMC de Conde e Monteiro', vislumbra-se a necessidade de definir o referencial mais adequado para investigações nacional ${ }^{2}$, objeto do presente estudo.

Em 2007, a OMS propôs um referencial de crescimento de cinco a 19 anos, publicando um 
novo conjunto de curvas elaboradas a partir de um referencial anterior, proposto em 2000 pelo CDC dos Estados Unidos, cujos dados foram revistos e reprocessados de maneira a atenuar os problemas anteriormente detectados com sua utilização. Essas modificações processadas pela OMS nos dados do CDC resultaram em um referencial que apresenta um ajuste bas-tante adequado. No Brasil os referenciais da OMS, além de serem recomendados por diversas sociedades científicas, também já vêm sendo utilizadas por instituições de saúde pública e privadas, o que enfatiza a necessidades de comparar as tendências de crescimento propostas pelas curvas da OMS com dados reais da população brasileira ${ }^{15-17}$.

Nota-se que há uma diversidade de critérios propostos para a avaliação do estado nutricional. Porém há uma grande dificuldade na determinação de um referencial internacional para o diagnóstico do estado nutricional e a diferença na composição corporal entre as populações mundiais. Diante dessa situação, realizou-se o presente estudo com o objetivo de avaliar o estado nutricional dos adolescentes, a partir dos critérios propostos por Conde e Monteiro ${ }^{1}$ e da $\mathrm{OMS}^{17}$, e comparar os diagnósticos obtidos.

\section{Métodos}

Trata-se de um estudo, transversal e analítico, com abordagem quantitativa, constituindo parte de um projeto ampliado, intitulado "Situação de Saúde no Maranhão", que estudou diversos indicadores de saúde da população maranhense, no período de julho de 2007 a janeiro de 2008, obtidos por conglomerados.

Utilizou-se um processo de amostragem aleatória sistemática de conglomerados, em estágios múltiplos, em três etapas. Na primeira, foram sorteados os municípios do Estado do Maranhão, de acordo com o último Censo Demográfico do Instituto Brasileiro de Geografia e Estatística $^{18}$, disponibilizado pela instituição. Foram sorteados 30 conglomerados. Em São Luís, capital do Estado, há três conglomerados computados no estudo. Os 27 municípios sorteados apresentam-se distribuídos aleatoriamente nas regiões geográficas do Estado.

No segundo estágio foram sorteados os setores censitários dentro de cada município, a partir de uma listagem dos setores, fornecida pelo IBGE. No terceiro estágio foi sorteado o ponto inicial dentro de cada setor, a partir do qual certo número de domicílios foi visitado.
Para o cálculo amostral, foi considerado como referência o evento mais relevante e que determinou um número amostral maior. Assim, a variável desnutrição foi o evento de referência para o cálculo de adolescente. Para tanto, considerouse o percentual de desnutrição no Brasil em 2003, da Pesquisa de Orçamento Familiar realizada pelo Instituto Brasileiro de Geografia e Estatística ${ }^{19}$ que foi de $8 \%$, resultando, então, no tamanho amostral de 1.256 adolescentes (incluídos 10\% de estimativa de perdas). Com este tamanho de amostra, o estudo foi capaz de avaliar os indicadores saúde dos adolescentes do Estado do Maranhão com uma margem de erro em geral inferior a $3 \%$; o efeito desenho foi 2 ; o intervalo de confiança 95\%; nível de significância de 5\% e precisão das estimativas de $3 \%$.

Como instrumento de coleta de dados, um questionário padronizado e pré-testado foi construído durante a pesquisa. As variáveis selecionadas correspondem às sociodemográficas e antropométricas: peso e altura.

O peso corporal foi medido em única tomada, utilizando-se balança digital portátil de precisão, com capacidade mínima de $2 \mathrm{~kg}$ e $100 \mathrm{~g}$ e máxima de $180 \mathrm{~kg}$ (Líder), concedida em caráter de empréstimo pelo Ministério da Saúde. A estatura foi verificada em tomada única utilizandose um estadiômetro portátil construído na pesquisa com escala de 0 a 200 centímetros e resolução de $0,1 \mathrm{~cm}$. O procedimento de coleta foi segundo as técnicas sugeridas por Jelliffe ${ }^{20}$.

A avaliação do estado nutricional foi realizada através da classificação da Organização Mundial de Saúde ${ }^{17}$ e da referência nacional de Valores Críticos de Conde e Monteiro ${ }^{1}$, visando traçar paralelo entre os índices encontrados nas duas curvas.

Para digitação e processamento de dados foi utilizado o programa Epi-Info do CDC de Atlanta, EUA ${ }^{21}$. Os testes qui-quadrado, concordância de McNemar e correlação de Speamar foram aplicados utilizando o programa SPSS for Windows 16.0.

Ressalta-se que a presente pesquisa foi submetida e aprovada pelo Comitê de Ética em Pesquisa do Hospital Universitário da Universidade Federal do Maranhão.

\section{Resultados}

Os resultados apresentados integram as análises dos dados relativos a 1.256 adolescentes. Quando comparado o IMC dos adolescentes nos dois 
critérios de classificação ${ }^{1,17}$, foi observado uma relação dependência do tipo de classificação daquele dos adolescentes do sexo masculino. No critério da OMS foram encontradas diferenças significativas em desnutrido e obeso e no de Conde e Monteiro em normal (peso adequado) e sobrepeso no sexo masculino. No sexo feminino não foi observada qualquer diferença significativa entre as classificações (Tabela 1).

Na Tabela 2, observa-se de um modo geral, que não há uma discordância significativa $(\mathrm{p}=$ $0,1637)$ entre os dois critérios.

Nota-se na Tabela 3 que há uma correlação significativa $(p=0,011)$ do estado nutricional entre as duas classificações.

\section{Discussão}

O peso, a altura e o IMC são medidas simples, confiáveis e amplamente utilizadas por profissionais de saúde pública e área clínica, por servirem como indicadores das condições de saúde da populaçãoo ${ }^{1,22}$.

A decisão sobre qual critério adotar para a classificação do estado nutricional em crianças e adolescentes a partir do IMC é ainda uma matéria em aberto ${ }^{3}$. Porém, é importante a investigação de valores de referência baseados em pesquisas nacionais, pois ampliaram os dados e as discussões, tanto no âmbito clínico como no epidemiológico.

Estudos revelam que a baixa sensibilidade dos pontos de cortes das referências americana e "internacional", nas quais chegam a identificar até $60 \%$ de adolescentes com excesso de gordura como eutróficos, podem retardar a busca de medidas preventivas efetivas ${ }^{2,14,23}$.
Silva et. al. ${ }^{7}$, no estudo realizado com os critérios sugeridos por Conde e Monteiro ${ }^{1}$ e Cole et al. ${ }^{11}$ observaram que não houve caso de baixo peso em ambos os sexos. Logo, de acordo com a OMS, ocorreu um caso de baixo peso.

Entretanto, no trabalho de Vitolo et al. ${ }^{14}$, os valores de referência de Conde e Monteiro, para população de adolescentes estudados mostrou maior sensibilidade para diagnosticar excesso de adiposidade quando comparado ao referencial internacional ${ }^{2}$.

No presente estudo foram encontradas diferenças significativas no diagnóstico nutricional para os adolescentes comparando os critérios de Conde e Monteiro ${ }^{1}$ e World Health Organization ${ }^{17}$ : normal (peso adequado) e sobrepeso; desnutrido e obeso, respectivamente, para o sexo masculino.

Farias Júnior et al. ${ }^{12}$ concluíram que os critérios de classificação do IMC (IOTF, CDC, WHO, Must et al. citado por Abrantes et al. ${ }^{13}$ ) estimaram de maneira similar a prevalência de excesso de peso, exceto os critérios propostos por Conde

Tabela 2. Distribuição de frequências de concordância e discordância, segundo a classificação da $\mathrm{OMS}^{17}$ e Conde e Monteiro ${ }^{1}$. Maranhão, 2007-2008.

\begin{tabular}{lccr}
\hline \multirow{2}{*}{ OMS } & \multicolumn{3}{c}{ Conde e Monteiro } \\
\cline { 2 - 4 } & Normal & Anormal & Total \\
\hline Normal & 1001 & 12 & 1013 \\
Anormal & 21 & 222 & 243 \\
Total & 1022 & 234 & 1256 \\
\hline $\mathrm{p}=0,1637$. Concordância $=97,4 \%$. Discordância $=2,6 \%$.
\end{tabular}

Tabela 1. Distribuição de frequência da classificação do IMC dos adolescentes de acordo com os critérios de IMC do $\mathrm{OMS}^{17}$ e de Conde e Monteiro'. Maranhão 2007-2008.

\begin{tabular}{|c|c|c|c|c|c|c|c|c|}
\hline \multirow{3}{*}{ IMC } & \multicolumn{4}{|c|}{ Masculino } & \multicolumn{4}{|c|}{ Feminino } \\
\hline & \multicolumn{2}{|c|}{ OMS } & \multicolumn{2}{|c|}{ Conde } & \multicolumn{2}{|c|}{ OMS } & \multicolumn{2}{|c|}{ Conde } \\
\hline & $\mathbf{n}$ & $\%$ & n & $\%$ & $\mathbf{n}$ & $\%$ & n & $\%$ \\
\hline Desnutrido & 26 & 6,1 & 13 & 3,1 & 29 & 3,5 & 38 & 4,6 \\
\hline Normal & 335 & 78,8 & 350 & 82,4 & 678 & 81,6 & 672 & 80,9 \\
\hline Sobrepeso & 45 & 10,6 & 52 & 12,2 & 107 & 12,9 & 104 & 12,5 \\
\hline Obeso & 19 & 4,5 & 10 & 2,4 & 17 & 2,0 & 17 & 2,0 \\
\hline \multirow[t]{2}{*}{ Total } & 425 & 100,0 & 425 & 100,0 & 831 & 100,0 & 831 & 100,0 \\
\hline & \multicolumn{4}{|c|}{$\chi^{2}=7,96 p=0,0468$} & \multicolumn{4}{|c|}{$\chi^{2}=1,27 p=0,7343$} \\
\hline
\end{tabular}


Tabela 3. Coeficiente de correlação de Spearmn do estado nutricional dos adolescentes, segundo entre os critérios de $\mathrm{OMS}^{17}$ e Conde e Monteiro ${ }^{1}$. Maranhão, 2007-2008.

\begin{tabular}{|c|c|c|c|c|c|}
\hline \multirow{2}{*}{ OMS } & \multicolumn{4}{|c|}{ Conde e Monteiro } & \multirow[b]{2}{*}{ Total } \\
\hline & Desnutrido & Normal & Sobrepeso & Obeso & \\
\hline Desnutrido & 42 & 13 & 0 & 0 & 55 \\
\hline Normal & 9 & 1001 & 3 & 0 & 1013 \\
\hline Sobrepeso & 0 & 8 & 143 & 1 & 152 \\
\hline Obeso & 0 & 0 & 10 & 26 & 36 \\
\hline Total & 51 & 1022 & 156 & 27 & 1256 \\
\hline
\end{tabular}

Correlação de Spearman $=0,934 \mathrm{p}=0,011$.

e Monteiro ${ }^{1}$, que resultaram em prevalência mais elevadas no sexo masculino, quadro que se configura com os resultados encontrados no presente estudo.

É necessário ressaltar um importante diferencial dos valores críticos para o IMC propostos por Conde e Monteiro ${ }^{1}$, que introduz valores referentes à desnutrição (baixo peso), que não estão presentes em outros estudos já bem definidos, limitando suas aplicações, uma vez que pode ser tão nociva à saúde quanto a própria obesidade ${ }^{24}$.

Abrantes et. al. ${ }^{13}$, empregando as propostas de Cole et al. ${ }^{11}$ e Must et al. citado por Abrantes et al. ${ }^{13}$, realizaram análises comparativas com 5.736 crianças e adolescentes brasileiros, e concluíram que a concordância entre os dois métodos foi satisfatória.

Os resultados da pesquisa de Silva et al. ${ }^{7}$ revelam que não houve diferença de diagnóstico entre os três critérios ${ }^{1,11,17}$, sendo verificado que para os meninos, as discordâncias evoluíram de forma sistemática com a idade.

No presente trabalho observou-se que não houve discordância significativa entre os critérios estudados. Houve uma concordância satisfatória $(97,4 \%)$.

No estudo realizado por Wang e Wang ${ }^{3}$, no qual foram empregadas as propostas de Cole et al. ${ }^{11}$ e Must et al. citado por Abrantes et al. ${ }^{13}$ foram encontradas concordâncias entre as duas classificações (estatística Kappa > 80). E uma correlação significativa entre os dois critérios, não foi encontrada na literatura que realizou a correlação entre dois ou mais critérios. Porém, encontraram-se estudos que realizaram a correlação com a obesidade e outro com atividade física.
Com essa análise pode-se perceber que não há muitas discordâncias entre os critérios utilizados, portanto não podemos apontar o melhor critério e as vantagens de se utilizar um ou outro. Contudo, vale ressaltar que o critério nacional também pode ser mais utilizado, já que não tem diferenças significativas com o preconizado pelo Ministério da Saúde, da OMS.

\section{Colaboradores}

ASV Costa, MEA Gama e MLB Martins participaram da concepção e desenho do trabalho; coleta de dados e análise interpretação de dados; MBC Chein e LMO Brito da concepção e desenho do trabalho; do planejamento e interpretação de dados; revisão crítica do conteúdo e aprovação da versão final do manuscrito. SR Tonial participou do planejamento e desenho do trabalho; coleta de dados e análise de dados; CLF Cunha da elaboração do rascunho; da concepção e desenho do trabalho; coleta de dados. DSM Silva e PRS Cruz participaram da análise de dados; revisão crítica do conteúdo; aprovação da versão final do manuscrito. 


\section{Referências}

1. Conde WL, Monteiro CA. Body mass index cutoff points for evaluation of nutritional status in Brazilian children and adolescents. J Pediatr 2006; 82(4):266-272

2. Kac G, Sichieri R, Gigante DP. Epidemiologia nutricional. Rio de Janeiro: Fiocruz; 2007.

3. Wang Y, Wang JQ. Standard definition of child overweight and obesity worldwide. Authors' standard compares well wil WHO standard. BMJ 2000; 321(7269):1158.

4. Araújo C, Toral N, Silva ACF, Velásquez-Melendez G, Dias AJR. Estado nutricional dos adolescentes e sua relação com variáveis sociodemográficas: Pesquisa Nacional de Saúde do Escolar (PeNSE), 2009. Cien Saude Colet 2010; 15(Supl. 2):3077-3084.

5. Rodrigues PA, Marques MH, Chaves MGAM, Souza CF, Carvalho MF. Prevalência e fatores associados a sobrepeso e obesidade em escolares da rede pública. Cien Saude Colet 2011; 16(Supl. 1):1581-1588.

6. Sigulem DM, Devincenzi MU, Lessa AC. Diagnosis of child and adolescent nutritional status. J Pediatr 2000; 76(3):275-284.

7. Silva HGV, Chiara VL, Barros ME, Rêgo AL, Ferreira A, Pitasi BA, Mattos T. Diagnóstico do estado nutricional de escolares: comparação entre critério nacional e internacional. J Pediatr 2008; 84(6): 550-555.

8. National Center for Health Statistics (NCHS). Growth Curves for Children, Birth-18 Years. Washington: NCHS; 1978.

9. Onis M, Victora CG. Gráficos de crescimento para bebês alimentados com leite materno. J Pediatr 2004; $80(4): 85-87$.

10. World Health Organization (WHO). Physical Status: the use and interpretation of anthropometry. Geneva: WHO; 1995.

11. Cole TJ, Bellizzi MC, Flegal KM, Dietz WH. Establishing a standard definition for child overweight and obesity worldwide: international survey. BMJ 2000; 320(7244):1240-1243.

12. Farias Júnior JC, Konrad LM, Rabacow FM, Grup S, Araújo VC. Sensibilidade e especificidade de critérios de classificação do índice de massa corporal em adolescentes. Rev Saude Publica 2009; 43(1):53-59.

13. Abrantes MM, Lamounier JA, Colosimo EA. Comparison of body mass index values proposed by Cole et al. (2000) and Must et al. (1991) for identifying obese children with weight-for-height index recommended by the World Health Organization. Public Health Nutrition. 2003; 6(03):307-311.

14. Vitolo MR, Campagnolo PDB, Barros ME, Gama CM, Ancona Lopez F. Avaliação de duas classificações para excesso de peso em adolescentes brasileiros. Rev Saude Publica 2007; 41(4):653-656.
15. Onis M, Onyango AW, Borghi E, Siyam A, Nishida C, Siekmann J. Development of a WHO growth reference for school-aged children and adolescents. Bull World Health Organ 2007; 85(9):660-667.

16. Vasconcelos VLd, Lapa TM, Carvalho EFd. Prevalência de sobrepeso e obesidade em adolescentes masculinos nas macrorregiões do Brasil, 1980-2000. Escola Anna Nery 2006; 10(3):417-424.

17. World Health Organization (WHO). Growth reference data for 5-19 years. [página na Internet] 2007. [acessado 2013 set 29]. Disponível em: http://www. who.int/growthref

18. Instituto Brasileiro de Geografia e Estatística (IBGE). Censo demográfico de 2000. [banco de dados na Internet] 2004. [acessado 2013 set 29]. Disponível em: http://www.ibge.gov.br/home/estatistica/populacao/default_censo_2000.shtm

19. Instituto Brasileiro de Geografia e Estatística (IBGE). Pesquisa de orçamentos familiares: 2002-2003: aquisição alimentar domiciliar per capita, Brasil e grandes regiões. Rio de Janeiro: IBGE; 2004.

20. Jellifee DB. The assessment of the nutritional status of the community. Geneva: World Health Organization (WHO); 1966.

21. Dean AG, Dean JA, Burton AH, Dicker RC. Epinfo, versão 6: um sistema de processamento de texto, banco de dados e estatística para computadores. São Paulo: Centers of Disease Control; 1994.

2. Garza C, De Onis M. A new international growth reference for young children. Am J Clin Nutr 1999; 70(1 Part 2):169-172.

23. Vieira ACR, Alvarez MM, Marins VMRd, Sichieri R, Veiga GVd. Desempenho de pontos de corte do índice de massa corporal de diferentes referências na predição de gordura corporal em adolescentes. Cad Saude Publica 2006; 22(8):1681-1690.

24. Fernandes RA, Segatto AFM, Silva CB, Oliveira AR, Freitas Junior IF. Estado nutricional de adolescentes segundo o índice de massa corporal. Motriz 2007; 13(2):106-113.

Artigo apresentado em 30/07/2012

Aprovado em 25/08/2012

Versão final apresentada em 02/09/2012 